\title{
Urinary nerve growth factor levels could be a biomarker for overactive bladder symptom: a meta-analysis
}

\author{
H.C. Qu, S. Yan, X.L. Zhang, X.W. Zhu, Y.L. Liu and P. Wang \\ Department of Urological Surgery, \\ The Fourth Affiliated Hospital of China Medical University, \\ Shenyang, Liaoning, China \\ Corresponding author: P. Wang \\ E-mail: cmu4h_wangping@126.com
}

Genet. Mol. Res. 13 (4): 8609-8619 (2014)

Received June 10, 2013

Accepted October 1, 2013

Published April 14, 2014

DOI http://dx.doi.org/10.4238/2014.April.14.14

\begin{abstract}
To examine whether urinary tract nerve growth factor (UNGF) could be a biomarker for overactive bladder (OAB) symptom, we conducted a comprehensive meta-analysis of 8 case-control studies. In all the studies considered, patients with $\mathrm{OAB}$ symptom had a higher uNGF level compared to healthy people. In addition, patients had a significantly lower UNGF level after successful treatment. In the subgroup analysis, we found that patients with OAB-wet symptom had a higher uNGF level than patients with OABdry symptom. However, no significant difference was found between patients with $\mathrm{OAB}$ symptom and patients with interstitial cystitis/painful bladder syndrome (IC/PBS) symptom in uNGF/Cr levels. In conclusion, uNGF level could be a useful biomarker for the diagnosis of OAB, a possible biomarker for differentiation between OAB subtypes (wet or dry), and a predictive biomarker for a specific treatment, but it cannot be used as the urinary biomarker for the differential diagnosis of IC/PBS and OAB.
\end{abstract}

Key words: Urinary nerve growth factor; Overactive bladder; Meta-analysis 


\section{INTRODUCTION}

Overactive bladder $(\mathrm{OAB})$ is a symptomatic diagnosis based on the presence of urgency, with or without incontinence, and is usually accompanied by frequency and nocturia. $\mathrm{OAB}$ has an overall prevalence in the adult population of above $10 \%$, but this may exceed $40 \%$ in elderly groups (Irwin et al., 2006). OAB symptom and interstitial cystitis/painful bladder syndrome (IC/PBS) are prevalent urologic diseases that can cause considerable morbidity (Jacobs et al., 2010). However, the term OAB does not actually identify specific causes or disease entities (Yokoyama et al., 2008). Although the use of an urgency perception scale or urgency severity score has been suggested, these instruments are based on subjective reporting by the patient who must grade the degree of urgency and may be difficult for patients to understand the term of urgency (De Wachter and Wyndaele, 2003; Nixon et al., 2005). One way to overcome this problem is to introduce an objective test for the diagnosis of OAB.

The term "biomarker" generally indicates anything that can be used as an indicator of a particular disease state, including its presence (diagnostic biomarker), severity, and/or its response to a specific treatment (predictive biomarker). Biomarkers can be specific cells, enzymes, hormones, genes, or gene products that can be detected and measured in parts of the body such as the blood, urine, or tissue (Bhide et al., 2013). Urinary tract nerve growth factor (UNGF) is produced by the bladder urothelium and smooth muscle (Steers et al., 1991). It has attracted considerable attention as a key player in the link between inflammation and altered pain signaling (Liu et al., 2011). NGF is responsible for the growth and maintenance of sensory neurons and appears to have a role in neuroimmune interactions, tissue inflammation, and neuroplasticity for neuronal events, leading to OAB. Increased expression of NGF is also present in bladder biopsies from women with sensory urgency or IC/PBS (Lowe et al., 1997). Many experimental data have reported increased level of UNGF in patients with OAB and IC/ PBS (Vijaya et al., 2013). We hypothesize that determination of uNGF level could serve as a valuable biomarker for the diagnosis of OAB. Furthermore, if uNGF levels can be reduced after successful therapy, the measurement of uNGF could be a useful objective tool for assessing the therapeutic outcome of OAB treatment (Liu et al., 2008). Therefore, we performed a meta-analysis by including the most recent and relevant articles to identify statistical evidence.

\section{MATERIAL AND METHODS}

\section{Literature search}

We performed an electronic search of the PubMed, Cochrane library, Embase, Web of Science, Springer Link, and CBM databases extensively to identify relevant studies available up to March 10, 2013. The search terms included ['overactive bladder' or 'OAB' (Mesh)] and ['nerve growth factor' or 'NGF' (Mesh)]. References in the eligible studies or textbooks were also reviewed through a manual search to identify other potentially eligible studies.

\section{Inclusion and exclusion criteria}

The included studies were required to meet the following criteria: i) the type of study should be a case-control study; ii) these case-control studies should focus on the association 
between the uNGF level and OAB symptom; iii) all patients were diagnosed with clinical OAB symptoms (confirmed by a 3-day voiding diary indicating a frequency of 8 episodes of urgency or 1 or more than 1 episodes of urgency incontinence per day); iv) patients were classified as OAB-wet or OAB-dry based on the voiding diary record (with or without urgency incontinence); v) the publication was in English. Studies were excluded if they reported incomplete, useless, or overlapping data; if they used median and interquartile ranges to describe the uNGF levels; or if they were meta-analyses, letters, reviews, or editorial articles.

\section{Data extraction}

Using a standardized form, data from published studies were independently extracted by two reviewers (H.C. Qu and S. Yan) to populate the necessary information. The following information was extracted from each of the articles: first author, year of publication, language, study design, source of cases and controls, number of cases and controls, mean age, sample, clinical symptom, treatment, and uNGF (or uNGF/Cr) level. In case of conflicting evaluations, an agreement was reached following a discussion with a third reviewer (P. Wang).

\section{Quality assessment of studies included}

Two reviewers (H.C. Qu and S. Yan) independently assessed the quality of papers according to modified STROBE quality score systems (von Elm et al., 2007; Zhang et al., 2011). Forty assessment items related to the quality appraisal were used in this meta-analysis, with scores ranging from 0 to 40 . Scores of $0-20,20-30$, and 30-40 were defined as low, moderate, and high quality. Disagreement was resolved by discussion.

\section{Statistical analysis}

The mean difference (MD) and 95\%CI were calculated using Review Manager Version 5.1.6 (provided by the Cochrane Collaboration, available at: http://ims.cochrane.org/revman/ download) and STATA Version 12.0 (StataCorp., College Station, TX, USA) software. Between-study variations and heterogeneities were estimated using Cochran's Q-statistic (Higgins and Thompson, 2002; Zintzaras and Ioannidis, 2005) ( $\mathrm{P} \leq 0.05$ was considered to be a manifestation of statistically significant heterogeneity). We also quantified the effect of heterogeneity by using the $\mathrm{I}^{2}$ test, which ranged from 0 to $100 \%$ and represented the proportion of inter-study variability that can be contributed to heterogeneity rather than by chance. When a significant Q-test $(\mathrm{P} \leq 0.05)$ or $\mathrm{I}^{2}>50 \%$ indicated that heterogeneity among studies existed, the randomeffect model was conducted for meta-analysis. Otherwise, the fixed-effect model was used. To establish the effect of heterogeneity on the conclusions of the meta-analyses, subgroup analysis was carried out. Funnel plots were often used to detect publication bias. However, because of the limitations caused by varied sample sizes and subjective reviews, the Egger linear regression test, which measured funnel plot asymmetry by using a natural logarithm scale of OR, was used to evaluate publication bias (Peters et al., 2006). When the $\mathrm{P}$ value was $<0.05$, publication bias was considered to be significant. All $\mathrm{P}$ values were two-sided. To ensure the reliability and the accuracy of the results, 2 reviewers (H.C. Qu and S. Yan) independently populated the data in the statistical software programs and obtained the same results. 


\section{RESULTS}

\section{Characteristics of studies included}

According to the inclusion criteria, 8 studies (Kim et al., 2006; Liu and Kuo, 2008, 2009; Liu et al., 2009; Kuo et al., 2010b; Chung et al., 2011; Oktar et al., 2013; Cho et al., 2013) met the inclusion criteria and were included in the meta-analysis. The flow chart of study selection is shown in Figure 1. The 8 case-control studies included 246 patients with OAB ( 80 patients were diagnosed as OAB-DRY, 102 were diagnosed as OAB-WET), 173 controls, and 148 patients with IC/PBS. Totally, 104 patients received successful treatment. The publication year of involved studies ranged from 2006 to 2013. All patients fulfilled the diagnostic criteria of clinical OAB symptoms (urgency or urgency incontinence) confirmed by a 3-day voiding diary (a frequency of 8 episodes of urgency or 1 or more than 1 episodes of urgency incontinence per day). The source of control samples were healthy people. All quality scores of included studies were more than 20 (moderate-high quality). The characteristics and methodological quality of the included studies are summarized in Table 1.

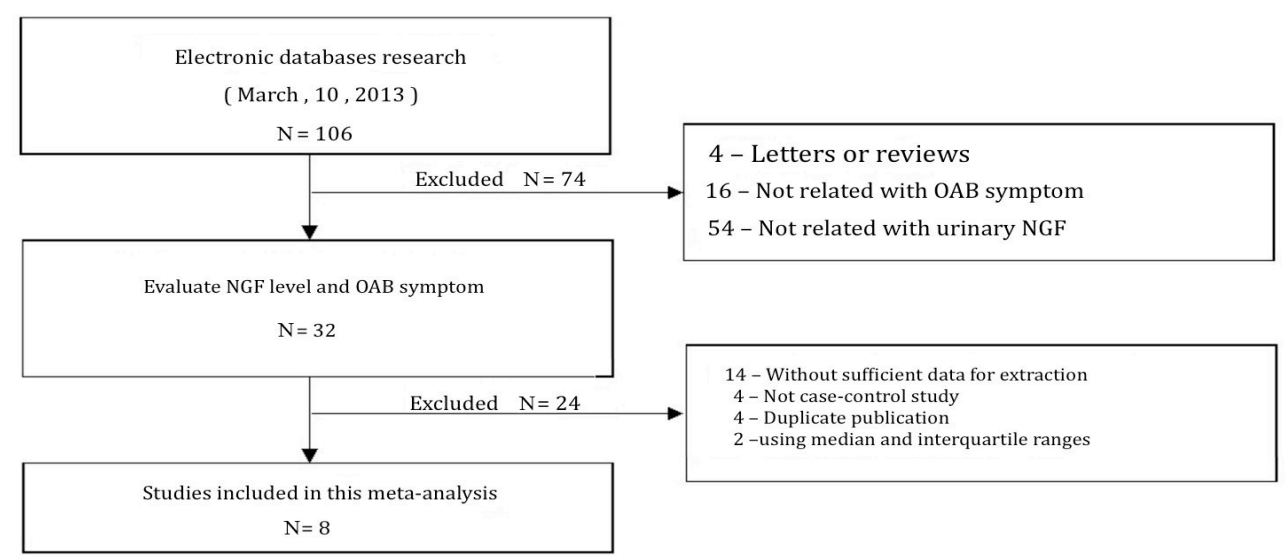

Figure 1. Flow chart shows study selection procedure. $\mathrm{NGF}=$ nerve growth factor; $\mathrm{OAB}=$ overactive bladder.

\begin{tabular}{|c|c|c|c|}
\hline Author (Year) & Method & Material $(\mathrm{N})$ & Quality score \\
\hline Liu et al. (2009) & $\mathrm{NGF}, \mathrm{NGF} / \mathrm{Cr}$ & Pre-treatment $(\mathrm{N}=50)$, post-treatment $(\mathrm{N}=50)$ & 25 \\
\hline Kuo et al. (2010b) & NGF, NGF/Cr & $\begin{array}{l}\text { 1. Wet }(\mathrm{N}=22) \text {, dry }(\mathrm{N}=26) \\
\text { 2. } \mathrm{OAB}(\mathrm{N}=22), \mathrm{IC} / \mathrm{PBS}(\mathrm{N}=60)\end{array}$ & 23 \\
\hline Kim et al. (2006) & NGF & $\mathrm{OAB}(\mathrm{N}=20)$, Control $(\mathrm{N}=65)$ & 25 \\
\hline Liu and Kuo (2009) & $\mathrm{NGF} / \mathrm{Cr}$ & $\mathrm{OAB}(\mathrm{N}=23), \mathrm{IC} / \mathrm{PBS}(\mathrm{N}=40)$ & 25 \\
\hline Chung et al. (2011) & NGF, NGF/Cr & $\begin{array}{l}\text { 1. } \mathrm{OAB}(\mathrm{N}=22), \text { Control }(\mathrm{N}=33) \\
\text { 2. } \mathrm{OAB}(\mathrm{N}=22), \mathrm{IC} / \mathrm{PBS}(\mathrm{N}=48)\end{array}$ & 26 \\
\hline Liu and Kuo (2008) & $\mathrm{NGF}, \mathrm{NGF} / \mathrm{Cr}$ & $\begin{array}{l}\text { 1. OAB }(\mathrm{N}=134) \text {, Control }(\mathrm{N}=40) \\
\text { 2. Wet }(\mathrm{N}=80) \text {, dry }(\mathrm{N}=54)\end{array}$ & 24 \\
\hline Oktar et al. (2013) & NGF, NGF/Cr & $\begin{array}{l}\text { 1. } \mathrm{OAB}(\mathrm{N}=40) \text {, Control }(\mathrm{N}=20) \\
\text { 2. Pre-treatment }(\mathrm{N}=40) \text {, post-treatment }(\mathrm{N}=40)\end{array}$ & 24 \\
\hline Cho et al. (2013) & NGF & $\begin{array}{l}\text { 1. } \mathrm{OAB}(\mathrm{N}=30) \text {, Control }(\mathrm{N}=15) \\
\text { 2. Pre-treatment }(\mathrm{N}=14), \text { post-treatment }(\mathrm{N}=14)\end{array}$ & 28 \\
\hline
\end{tabular}

$\mathrm{NGF}=$ nerve growth factor; $\mathrm{OAB}=$ overactive bladder; IC/PBS = interstitial cystitis/painful bladder syndrome. 


\section{Difference in the uNGF levels of patients with OAB symptom and healthy subjects}

A summary of the meta-analysis findings of the difference in the UNGF levels between patients with $\mathrm{OAB}$ symptom and healthy subjects is provided in Figure 2. The meta-analysis showed that patients with OAB symptom had a higher uNGF level than healthy people $(\mathrm{MD}=31.74,95 \% \mathrm{CI}=9.48-54.00, \mathrm{P}=0.005)$. Sensitivity analysis was conducted by omitting single studies, and no influence was found on the significance of the pooled MD.

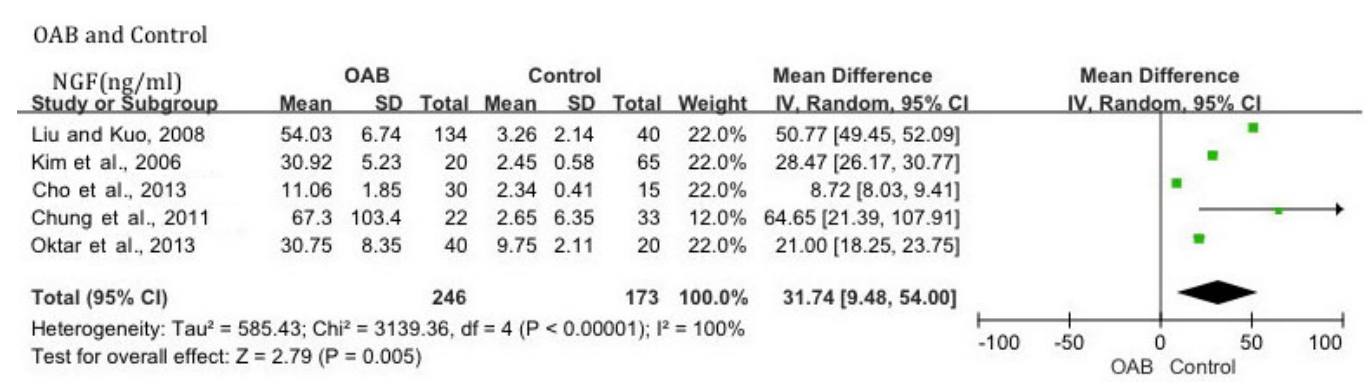

Figure 2. Meta-analysis of the difference between patients with OAB symptom and healthy controls (in uNGF levels).

\section{Difference in the uNGF levels or uNGF/Cr levels (uNGF level was normalized to its urine Cr level) of patients with OAB-dry and OAB-wet symptoms (uNGF level was normalized to its urine $\mathrm{Cr}$ level)}

The difference between patients with OAB-dry and OAB-wet symptoms is provided in Figures 3 and 4. The meta-analysis showed that patients with OAB-wet symptom had a higher $\mathrm{uNGF}$ or $\mathrm{uNGF} / \mathrm{Cr}$ level than patients with $\mathrm{OAB}$-dry symptom $(\mathrm{MD}=46.49,95 \% \mathrm{CI}$ $=43.94-49.05, \mathrm{P}<0.00001$ or $\mathrm{MD}=1.32,95 \% \mathrm{CI}=1.26-1.38, \mathrm{P}<0.00001)$. Sensitivity analysis was conducted by omitting single studies, and no influence was found on the significance of the pooled MD.

OAB-DRY and OAB-WET

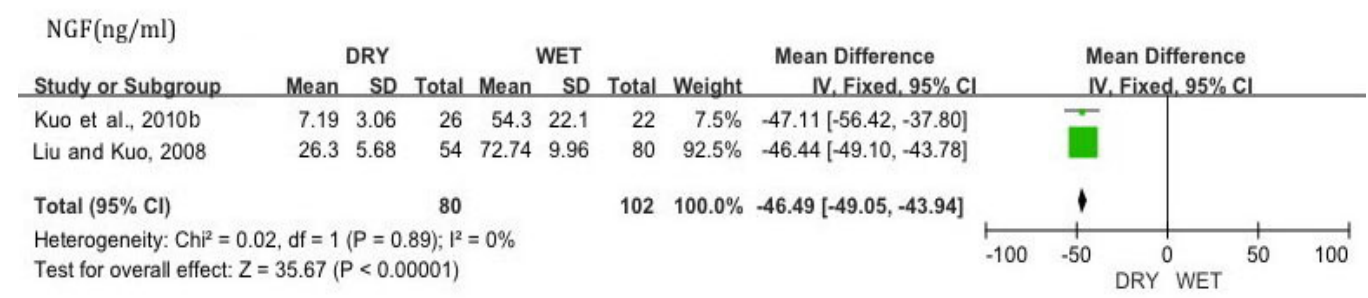

Figure 3. Meta-analysis of the difference between patients with OAB-dry symptom and OAB-wet symptom (in uNGF levels). 


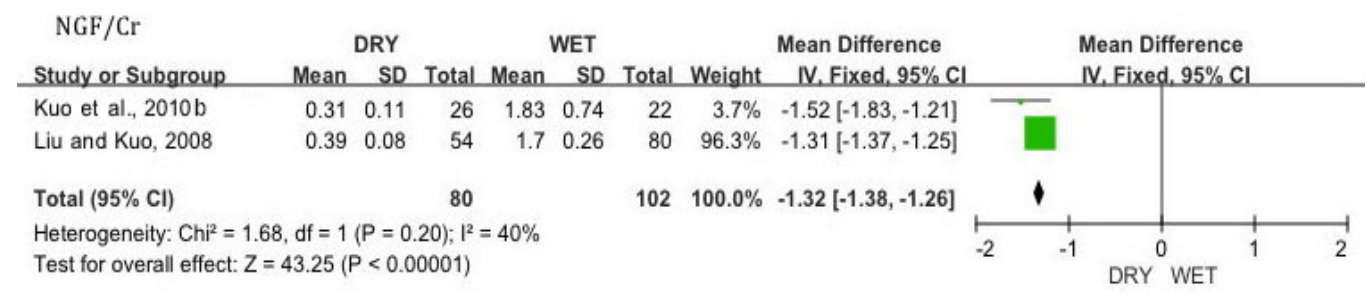

Figure 4. Meta-analysis of the difference between patients with OAB-dry symptom and OAB-wet symptom (in $\mathrm{uNGF/Cr} \mathrm{levels)}$

\section{Difference in the uNGF/Cr level of patients with OAB symptom and IC/PBS symptom}

The meta-analysis showed no significant difference in the $\mathrm{uNGF/Cr}$ level between patients with $\mathrm{OAB}$ symptom and those with $\mathrm{IC} / \mathrm{PBS}$ symptom $(\mathrm{P}=0.08$; Figure 5$)$. Sensitivity analysis was conducted by omitting single studies, and no influence was found on the significance of the pooled MD.

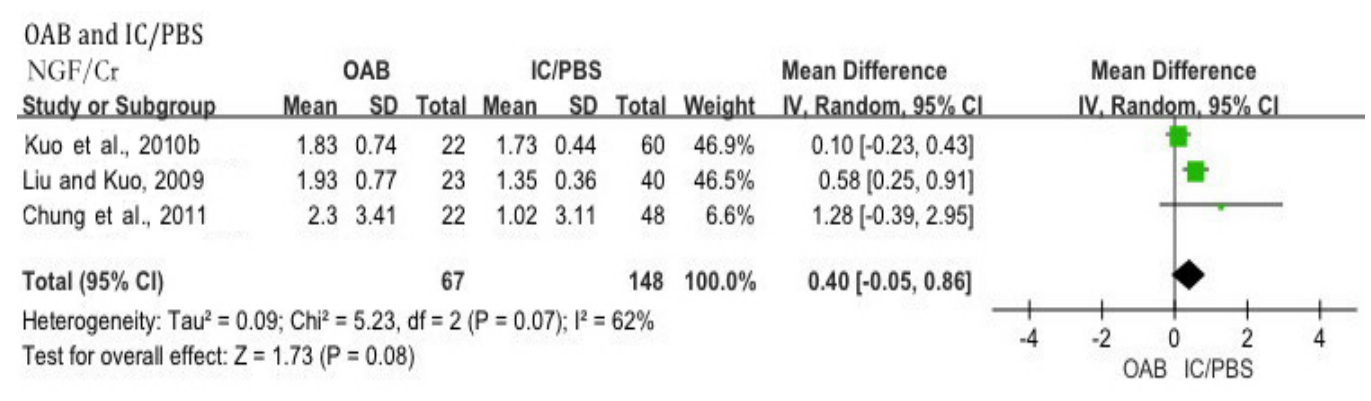

Figure 5. Meta-analysis of the difference between patients with OAB symptom and patients with IC/PBS symptom (in $\mathrm{uNGF/Cr}$ level).

\section{Difference in the uNGF levels or uNGF/Cr levels of patients with OAB symptom before and after successful treatment}

A summary of the meta-analysis findings of the difference in the UNGF levels or $\mathrm{uNGF} / \mathrm{Cr}$ levels of patients with OAB symptom before and after successful treatment is provided in Figures 6 and 7. The meta-analysis showed that patients have a significantly lower $\mathrm{uNGF}$ or $\mathrm{uNGF} / \mathrm{Cr}$ level after successful $\mathrm{OAB}$ treatment $(\mathrm{MD}=11.80,95 \% \mathrm{CI}=$ 4.61-19.00, $\mathrm{P}=0.001$ or $\mathrm{MD}=0.53,95 \% \mathrm{CI}=0.21-0.84, \mathrm{P}=0.001)$. Sensitivity analysis was conducted by omitting single studies, and no influence was found on the significance of the pooled MD. 
Pre-treatment and Post-treatment $\mathrm{NGF}(\mathrm{ng} / \mathrm{ml})$

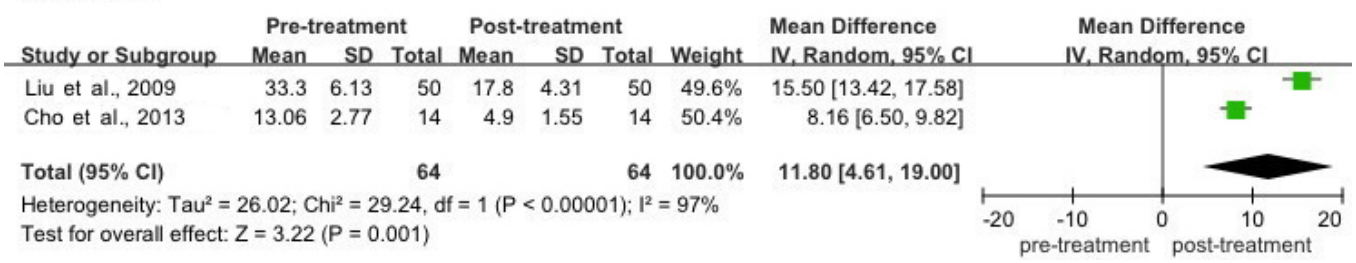

Figure 6. Meta-analysis of the difference between patients with $\mathrm{OAB}$ symptom before and after successful treatment (in uNGF levels).

Pre-treatment and Post-treatment

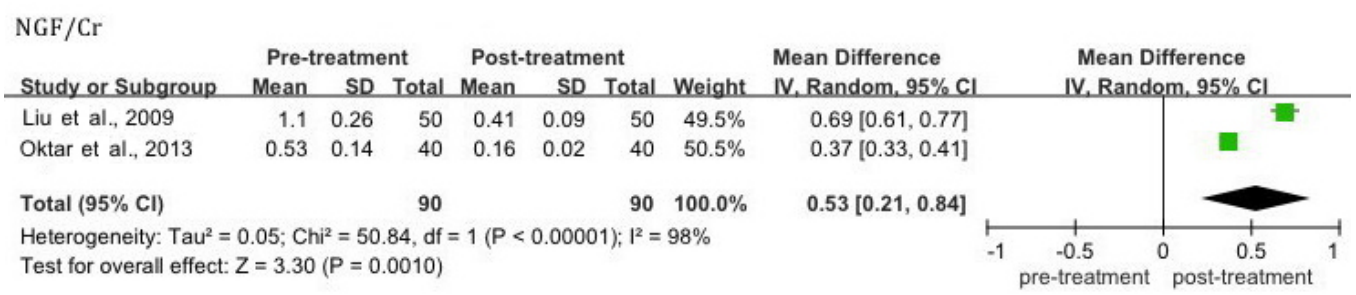

Figure 7. Meta-analysis of the difference between patients with $\mathrm{OAB}$ symptom before and after successful treatment (in $\mathrm{uNGF} / \mathrm{Cr}$ level).

\section{Publication bias}

Publication bias of the literature was assessed by Begger's funnel plot and the Egger linear regression test. The Egger linear regression test was used to measure the asymmetry of the funnel plot. All graphical funnel plots of the studies included appeared to be symmetrical (Figures 8-11). The Egger test also showed that there was no publication bias.

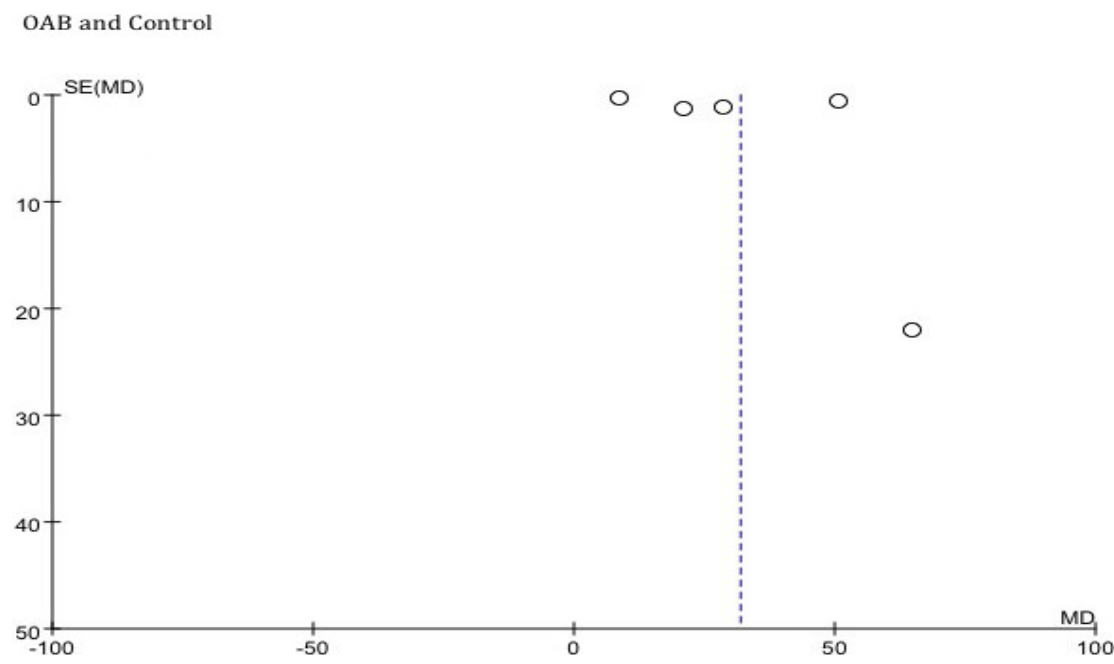

Figure 8. Begger's funnel plot of publication bias. 
H.C. Qu et al.

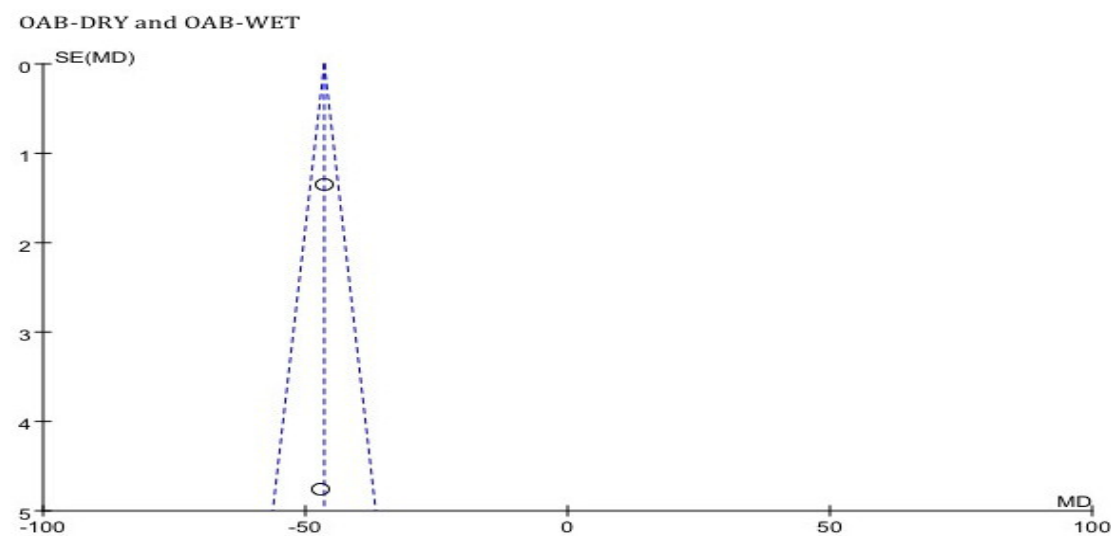

Figure 9. Begger's funnel plot of publication bias.

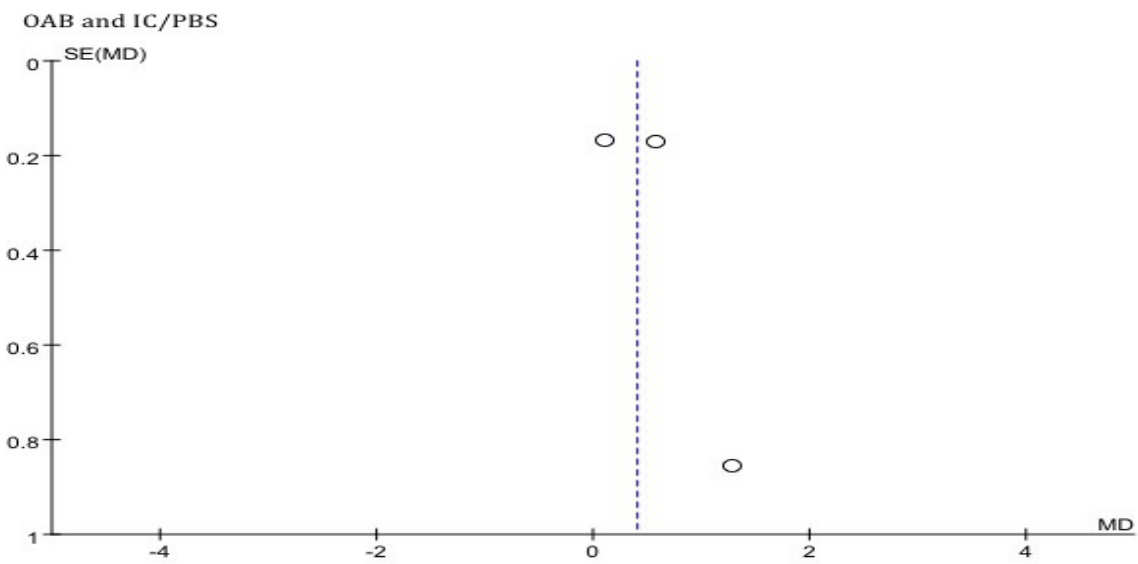

Figure 10. Begger's funnel plot of publication bias.

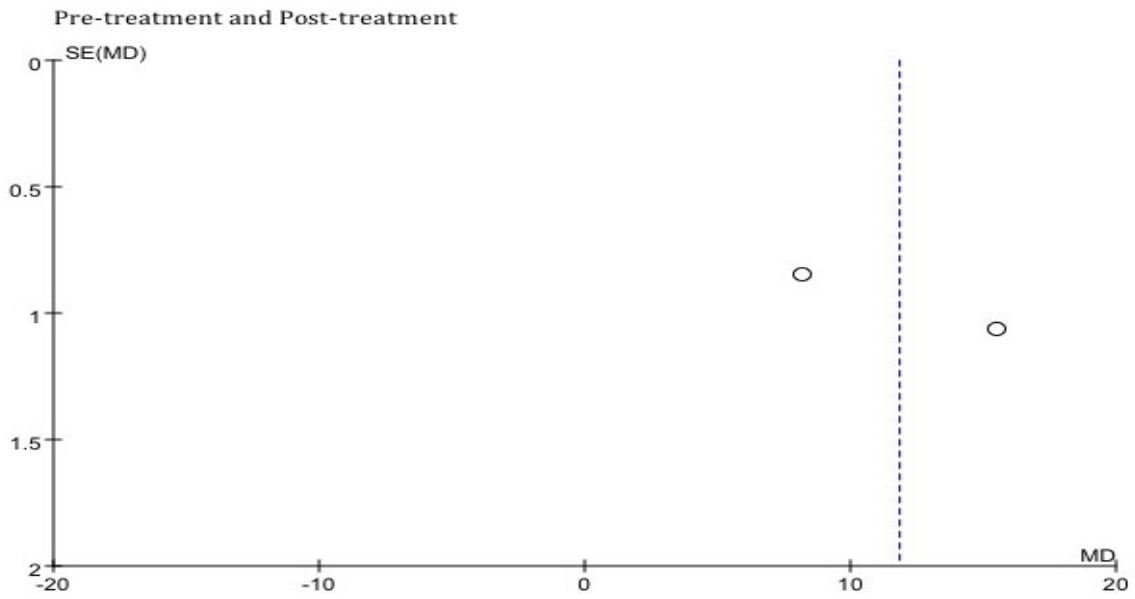

Figure 11. Begger's funnel plot of publication bias. 


\section{DISCUSSION}

$\mathrm{OAB}$ is a symptomatic diagnosis based on the presence of urgency, with or without incontinence. It has an overall prevalence in the adult population (Irwin et al., 2006). Although the pathophysiology of $\mathrm{OAB}$ has not been fully understood, $\mathrm{OAB}$ is partially caused by changes in the afferent nerves, and NGF plays an important role in this process (Kim et al., 2006).

Produced by the urothelium and bladder muscles, NGF regulates nerve cell growth and survival, and induces the sensitization of nociceptive sensory neurons (Kim et al., 2006). Recent studies showed that NGF provides mechanisms for bidirectional communication between the muscle or urothelium and nerve by triggering morphological and physiological alterations in the afferent and efferent neurons, leading to OAB symptom (Steers, 2002; Kim et al., 2006; Liu an Kuo, 2009). Bladder outlet obstruction in rats has been shown to cause an alteration in the urinary NGF levels (Chul et al., 2001) and it has been found to be associated with OAB and other lower urinary tract dysfunctions such as interstitial cystitis and bladder cancer (Liu and Kuo, 2009). Another study showed that urinary NGF levels decreased with the decrease in the severity of urgency and increased when the OAB symptom recurred (Liu et al., 2009).

In our meta-analysis, we included 8 independent studies to examine the association of urinary NGF level with OAB symptom. Overall, our analysis showed that patients with OAB symptom had a higher uNGF level than healthy people. This result suggested that uNGF levels could be a potential biomarker for the diagnosis of OAB. In addition, we found that UNGF decreased in response to effective treatment [antimuscarinic therapy (Liu et al., 2009) and anticholinergic therapy (Cho et al., 2013)], showing that uNGF level could be used to assess the therapeutic effects of $\mathrm{OAB}$ treatment. Interestingly, we even identified that patients with OAB-wet had significantly higher uNGF levels than those with OAB-dry. Previous studies provided evidence that patients with OAB-wet had higher uNGF/Cr levels than those with OAB-dry (Digesu et al., 2003; Hashim and Abrams, 2006; Liu et al., 2010; Kuo et al., 2010a). A possible reason for the difference in the uNGF levels between OAB-dry and OAB-wet could be the different degrees of inflammation and a higher percentage of DO (detrusor overactivity) in patients with OAB-wet. Hyman et al. (2001) found a higher incidence of DO associated with urgency incontinence (OAB-wet) than with the symptoms of urgency and frequency or nocturia or difficult urination (75 vs 36\%). Hashim and Abrams (2006) also found that $69 \%$ of men and $44 \%$ of women with OAB-dry had DO, while $90 \%$ of men and $58 \%$ of women with OAB-wet had DO. In another group of 843 women classified as having OAB, 457 (54.2\%) had urodynamically proven DO (Digesu et al., 2003). Therefore, uNGF could be a possible biomarker for differentiation between OAB subtypes. However, we found no significant difference between patients with OAB symptom and patients with IC/PBS symptom in UNGF/ $\mathrm{Cr}$ levels. The clinical symptoms of IC/PBS and OAB are similar, except that bladder pain typically presents in IC/PBS, and the urgency or urge incontinence presents in patients with OAB-dry and OAB-wet. Recent research has revealed that uNGF levels increase in both patients with IC/PBS and patients with OAB symptom (Liu et al., 2008, 2010). Thus, we need to find other urinary biomarkers for the differential diagnosis of IC/PBS and OAB.

Similar to other meta-analyses, this study has many limitations. First, the relevant research articles were not many and the sample size of this meta-analysis was not large. In addition, some relevant studies could not be included in our analysis because of incomplete raw data. Third, we could not address the sources of heterogeneity among all studies. Fourth, although all cases and controls of each study were well defined with similar inclusion criteria, 
there might be potential factors that were not taken into account and they might influence our result. Fifth, meta-analysis is retrospective research that is subject to methodological limitations. Most important of all, our meta-analysis was based on unadjusted MD estimates because not all published studies presented adjusted MDs or presented MDs, which were not adjusted by the same potential confounders, for example, age, ethnicity, and timing of urine collection. Given these results, additional investigations in these areas are needed, and our conclusions should be interpreted cautiously.

In conclusion, this meta-analysis of 8 case-control studies demonstrates that uNGF (or $\mathrm{uNGF} / \mathrm{Cr}$ ) level could be a useful biomarker for the diagnosis of $\mathrm{OAB}$, a possible biomarker for differentiation between $\mathrm{OAB}$ subtypes (wet or dry), and a predictive biomarker for a specific treatment. However, it cannot be the urinary biomarker for the differential diagnosis of IC/PBS and OAB. Because of the limited number of published studies in this field, the current available evidence remains limited. Therefore, we emphasize the necessity of conducting large studies with adequate methodological quality and proper control of confounds to obtain valid results.

\section{REFERENCES}

Bhide AA, Cartwright R, Khullar V and Digesu GA (2013). Biomarkers in overactive bladder. Int. Urogynecol. J. 24: 1065-1072.

Cho KJ, Kim HS, Koh JS and Kim JC (2013). Changes in urinary nerve growth factor and prostaglandin E(2) in women with overactive bladder after anticholinergics. Int. Urogynecol. J. 24: 325-330.

Chul KJ, Il SS, Hyun PY and Kon Hwang TA (2001). Changes in detrusor and urinary growth factors according to detrusor function after partial bladder outlet obstruction in the rat. Urology 57: 371-375.

Chung SD, Liu HT, Lin H and Kuo HC (2011). Elevation of serum C-reactive protein in patients with OAB and IC/BPS implies chronic inflammation in the urinary bladder. Neurourol. Urodyn. 30: 417-420.

De Wachter S and Wyndaele JJ (2003). Frequency-volume charts: a tool to evaluate bladder sensation. Neurourol. Urodyn. 22: 638-642.

Digesu GA, Khullar V, Cardozo L and Salvatore S (2003). Overactive bladder symptoms: do we need urodynamics? Neurourol. Urodyn. 22: 105-108.

Hashim H and Abrams P (2006). Is the bladder a reliable witness for predicting detrusor overactivity? J. Urol. 175: 191-194.

Higgins JP and Thompson SG (2002). Quantifying heterogeneity in a meta-analysis. Stat. Med. 21: 1539-1558.

Hyman MJ, Groutz A and Blaivas JG (2001). Detrusor instability in men: correlation of lower urinary tract symptoms with urodynamic findings. J. Urol. 166: 550-552.

Irwin DE, Milsom I, Hunskaar S, Reilly K, et al. (2006). Population-based survey of urinary incontinence, overactive bladder, and other lower urinary tract symptoms in five countries: results of the EPIC study. Eur. Urol. 50: 1306-1314.

Jacobs BL, Smaldone MC, Tyagi V, Philips BJ, et al. (2010). Increased nerve growth factor in neurogenic overactive bladder and interstitial cystitis patients. Can. J. Urol. 17: 4989-4994.

Kim JC, Park EY, Seo SI, Park YH, et al. (2006). Nerve growth factor and prostaglandins in the urine of female patients with overactive bladder. J. Urol. 175: 1773-1776.

Kuo HC, Liu HT, Tyagi P and Chancellor MB (2010a). Urinary nerve growth factor levels in urinary tract diseases with or without frequency urgency symptoms. LUTS 2: 88-94.

Kuo HC, Liu HT and Chancellor MB (2010b). Urinary nerve growth factor is a better biomarker than detrusor wall thickness for the assessment of overactive bladder with incontinence. Neurourol. Urodyn. 29: 482-487.

Liu HT and Kuo HC (2008). Urinary nerve growth factor level could be a potential biomarker for diagnosis of overactive bladder. J. Urol. 179: 2270-2274.

Liu HT and Kuo HC (2009). Urinary nerve growth factor levels are elevated in patients with overactive bladder and do not significantly increase with bladder distention. Neurourol. Urodyn. 28: 78-81.

Liu HT, Kuo HC and Chancellor MB (2008). The urinary nerve growth factor levels decrease with time in association with resolution of urgency severity after antimuscarinic therapy in patients with overactive bladder. BJU Int. 102: $1440-1444$. 
Liu HT, Chancellor MB and Kuo HC (2009). Decrease of urinary nerve growth factor levels after antimuscarinic therapy in patients with overactive bladder. BJU Int. 103: 1668-1672.

Liu HT, Chen CY and Kuo HC (2010). Urinary nerve growth factor levels in overactive bladder syndrome and lower urinary tract disorders. J. Formos. Med. Assoc. 109: 862-878.

Liu HT, Lin H and Kuo HC (2011). Increased serum nerve growth factor levels in patients with overactive bladder syndrome refractory to antimuscarinic therapy. Neurourol. Urodyn. 30: 1525-1529.

Lowe EM, Anand P, Terenghi G, Williams-Chestnut RE, et al. (1997). Increased nerve growth factor levels in the urinary bladder of women with idiopathic sensory urgency and interstitial cystitis. Br. J. Urol. 79: 572-577.

Nixon A, Colman S, Sabounjian L, Sandage B, et al. (2005). A validated patient reported measure of urinary urgency severity in overactive bladder for use in clinical trials. J. Urol. 174: 604-607.

Oktar T, Kocak T, Oner-Iyidogan Y, Erdem S, et al. (2013). Urinary nerve growth factor in children with overactive bladder: A promising, noninvasive and objective biomarker. J. Pediatr. Urol. 9: 617-621.

Peters JL, Sutton AJ, Jones DR, Abrams KR, et al. (2006). Comparison of two methods to detect publication bias in metaanalysis. JAMA 295: 676-680.

Steers WD (2002). Pathophysiology of overactive bladder and urge urinary incontinence. Rev. Urol. 4 (Suppl 4): S7-S18.

Steers WD, Kolbeck S, Creedon D and Tuttle JB (1991). Nerve growth factor in the urinary bladder of the adult regulates neuronal form and function. J. Clin. Invest. 88: 1709-1715.

Vijaya G, Cartwright R, Derpapas A, Gallo P, et al. (2013). Changes in nerve growth factor level and symptom severity following antibiotic treatment for refractory overactive bladder. Int. Urogynecol. J. 24: 1523-1528.

von Elm E, Altman DG, Egger M, Pocock SJ, et al. (2007). The Strengthening the Reporting of Observational Studies in Epidemiology (STROBE) statement: guidelines for reporting observational studies. Epidemiology 18: 800-804.

Yokoyama T, Kumon H and Nagai A (2008). Correlation of urinary nerve growth factor level with pathogenesis of overactive bladder. Neurourol. Urodyn. 27: 417-420.

Zhang L, Liu JL, Zhang YJ and Wang H (2011). Association between HLA-B*27 polymorphisms and ankylosing spondylitis in Han populations: a meta-analysis. Clin. Exp. Rheumatol. 29: 285-292.

Zintzaras E and Ioannidis JP (2005). Heterogeneity testing in meta-analysis of genome searches. Genet. Epidemiol. 28: 123-137. 